\title{
Superconducting re-entrant cavity transducer for a resonant bar gravitational radiation antenna
}

\author{
N. P. Linthorne and D. G. Blair \\ Department of Physics, University of Western Australia, Nedlands, Western Australia 6009, Australia
}

(Received 30 March 1992; accepted for publication 11 May 1992)

\begin{abstract}
A $10-\mathrm{GHz}$ superconducting niobium re-entrant cavity parametric transducer was developed for use in a cryogenic 1.5-tonne $\mathrm{Nb}$ resonant bar gravitational radiation antenna. The transducer has a very high electrical $Q\left(6 \times 10^{5}\right.$ at $\left.4.2 \mathrm{~K}\right)$, and was operated at high cavity fields without degrading the $Q$. A very high electromechanical coupling between the antenna and the transducer was therefore achieved. The highest coupling attained, constrained by the available pump power, was 0.11 . If the transducer were to be opcrated in conjunction with a wideband impedance matching element, an antenna bandwidth comparable to the frequency of the antenna would be attained. The temperature dependence of the $Q$ of the transducer was in good agreement with theory. At temperatures above about $6 \mathrm{~K}$ the $Q$ was degraded by the increase in the BCS surface resistance, while at lower temperatures the $Q$ was limited by radiative losses.
\end{abstract}

\section{INTRODUCTION}

Many physics experiments to detect weak forces involve the detection of small vibrations. One extreme example is the search for gravitational radiation using a cryogenic resonant bar antenna. To be able to detect bursts of gravitational radiation from energetic astrophysical sources in our galaxy, the transducer for the antenna must be capable of detecting a change in the vibration amplitude of the bar of less than $\sim 10^{-18} \mathrm{~m}$, with a resolution time of less than a few seconds. A microwave re-entrant cavity parametric transducer is well suited to this application. ${ }^{1-4}$ This type of transducer may have high sensitivity, small backaction, and a total noise level that approaches the quantum limit.

The gravitational radiation research group at the University of Western Australia has developed a niobium capacitance-modulated re-entrant cavity parametric transducer that operates at around $9.8 \mathrm{GHz}$ (microwave $X$ band). The transducer is used in a cryogenic 1.5-tonne $\mathrm{Nb}$ antenna. A schematic representation of the antenna and transducer system is shown in Fig. 1. The antenna consists of a 1.5-tonne cylindrical niobium bar that is supported by a low acoustic loss, high isolation mechanical filter. ${ }^{5}$ The bar has a very high acoustic $Q$ factor $\left(2.3 \times 10^{8}\right)$ in its fundamental longitudinal mode $(700 \mathrm{~Hz})$. A resonant niobium bending flap, tuned to the bar's fundamental longitudinal mode, is bonded to one end of the bar, and the vibration amplitude of the bending flap is monitored with a re-entrant cavity transducer that is also bonded to the antenna. The bending flap acts as a mechanical transformer, to match the high output impedance of the bar to the low mechanical input impedance of the transducer.

The re-entrant cavity transducer is basically an LC resonator, where the capacitance is determined by the spacing between the central post of the cavity and the bending flap, and the inductance is mainly due to the central section of the cavity. Relative motion of the cavity and the bending flap modulates the capacitance of the cavity, causing phase modulation of the pump signal, and produc- ing sidebands which are displaced from the pump by the antenna frequency. The carrier reflected from the cavity is suppressed relative to the sidebands to prevent saturation of the low-noise amplifier. A double-balanced mixer is used to recover the signal at the antenna frequency, using part of the original pump signal as the reference. High transducer sensitivity is obtained by using a small value of the capacitance gap $(\sim 10 \mu \mathrm{m})$, and a superconducting niobium cavity to reduce electrical losses. The transducer is pumped by an ultralow phase noise signal that is transmitted to the cavity by a thin coaxial cable (UT20). Operating the cavity in reflection allows just a single coaxial cable to be used. The coaxial cable is bonded to a low-loss three-stage mechanical filter to reduce vibrational coupling from the experimental chamber to the antenna.

At present, the detection sensitivity and bandwidth of the antenna are limited by the electromechanical coupling, $\beta$, between the antenna and the transducer. For a parametric re-entrant cavity transducer, the coupling is proportional to $Q_{0}{ }^{2} P_{i}$, where $Q_{0}$ is the unloaded electrical $Q$ of the cavity, and $P_{i}$ is the input power to the cavity. In this paper it is shown that the re-entrant cavity transducer can have a very high electrical $Q\left(>6 \times 10^{5}\right.$ at $\left.4.2 \mathrm{~K}\right)$, and that the transducer can be operated at high cavity fields without degrading the $Q$. A very high electromechanical coupling was achieved with this transducer. The highest value reached was $\beta \approx 0.11$.

The equations governing the electrical losses in the transducer are also presented. The temperature dependence of the cavity $Q$ was in good agreement with theory. At temperatures above about $6 \mathrm{~K}$ the $Q$ was degraded by the increase in the BCS surface resistance, while at lower temperatures the $Q$ was limited by radiative losses.

\section{DESCRIPTION OF THE RE-ENTRANT CAVITY TRANSDUCER}

Figure 2 shows the bonded re-entrant cavity transducer in detail. The bonded transducer is similar to the levitated re-entrant cavity transducer ${ }^{6,7}$ and includes a base 


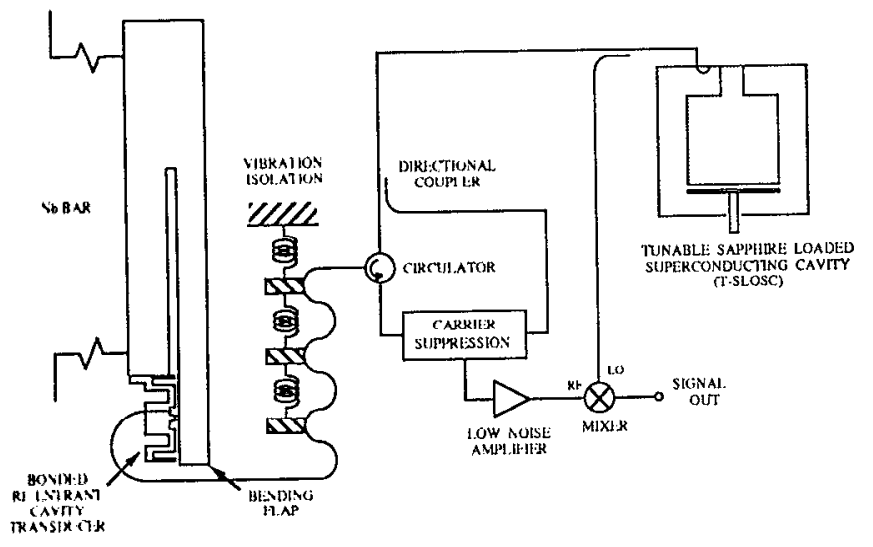

FIG. 1. Schematic diagram of the cryogenic 1.5-tonne $\mathrm{Nb}$ antenna with a bonded bending flap and bonded re-entrant cavity transducer.

so that it may be attached to the antenna. The central resonant section of the transducer is surrounded by an if choke to reduce radiative losses. The central post, which protrudes $0.05 \mu \mathrm{m}$ past the top face of the cavity, has slightly rounded edges to prevent premature electric field breakdown, and the top face of the cavity has a $0.3^{\circ}$ taper to allow for small misalignments of the cavity with the sense face of the bending flap. The gap between the end of the post and the bending flap is about $13 \mu \mathrm{m}$ when the resonant frequency of the cavity is $9.8 \mathrm{GHz}$. The cavity dimensions are such that the only allowable resonant mode is transverse electromagnetic (TEM). Radial TE and TM modes are prohibited by the choosing the depth of the cavity to be $\ell<\lambda_{0} / 4$, and the outer radius of the cavity to be $r_{2}<\lambda_{0} / 3.41$, where $\lambda_{0}$ is the freespace wavelength (3.1 $\mathrm{cm}$ at $9.8 \mathrm{GHz}$ ). At resonance, the field configuration consists mainly of a circumferential magnetic field encircling the re-entrant post, and an axial electrical field between the end of the post and the sense face of the bending flap.

The resonant frequency of the cavity may be calculated using the standard formulas for the capacitance and inductance of a coaxial line. ${ }^{8}$ At $9.8 \mathrm{GHz}$ the cavity has a calculated total capacitance of $C_{0}=0.43 \mathrm{pF}$, an inductance of $L_{0}=0.61 \mathrm{nH}$, and a resonant gap spacing of $x_{0 r}=13.0$

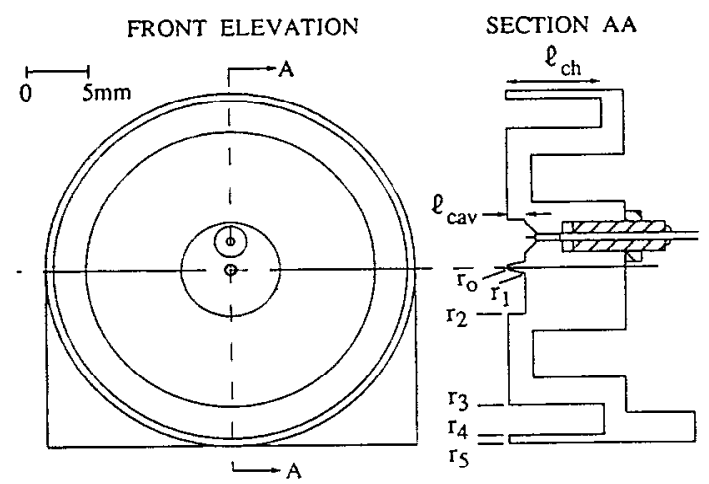

FIG. 2. Scale drawing of the bonded re-entrant cavity transducer. Also shown is the microwave coupling probe. The transducer dimensions are $r_{0}=0.40 \mathrm{~mm}, r_{1}=0.50 \mathrm{~mm}, r_{2}=4.00 \mathrm{~mm}, r_{3}=11.75 \mathrm{~mm}, r_{4}=14.35 \mathrm{~mm}$, $r_{\mathrm{s}}=15.00 \mathrm{~mm}, \ell=1.40 \mathrm{~mm}, \ell_{\mathrm{cav}} 1.35 \mathrm{~mm}$, and $\ell_{\mathrm{ch}}=7.60 \mathrm{~mm}$. $\mu \mathrm{m}$. The dynamic (or effective) gap spacing, which is given by $x_{0}=-C_{0} /\left(d C_{0} / d x_{0 r}\right)$, is $x_{0}=15.7 \mu \mathrm{m}$.

Prior to bonding the cavity to the antenna, the surface of the cavity and the sense face of the bending flap were chemically etched in a hydrofluoric and nitric acid solution. This procedure removed a machine damaged layer of around $100 \mu \mathrm{m}$, and is a reliable method for producing a high electrical $Q$ in a re-entrant cavity. Immediately after polishing, the cavity was subjected to anodic oxidation. This produces a protective film a few hundred nanometers thick which prevents $Q$ degradation even if the cavity is exposed to air. Kolesov et al. ${ }^{9}$ found that higher breakdown fields could be attained in an anodized re-entrant cavity than in an unanodized cavity because the dielectric cffect of the oxide layer reduces the effective field strength at surface peaks in the cavity.

Coupling to the cavity was achieved using an electric field probe inserted about halfway across the bottom of the cavity. The probe was formed by a UT20 semirigid coaxial cable that was soldered to a threaded stainless-steel stud. The coupling was adjusted by varying the position of the stud in the cavity. After soldering the coaxial cable to the stud and exposing the Tefion and center conductor to make the probe, the assembly was thermally cycled between 300 and $77 \mathrm{~K}$ several times. This minimized retraction of the Teflon down the outer conductor during subsequent cooling of the cavity. The end of the probe was rounded with fine abrasive powder to minimize the risk of electrical breakdown of the probe. The coupling hole in the cavity was countersunk to reduce the sensitivity of the coupling to the variation in the probe position, and so make the coupling easier to adjust.

Ideally, the microwave coupling to the transducer should be set to unity so as to provide the greatest level of intrinsic carrier suppression. The coupling was sct at room temperature, allowing for the increase in the transducer $Q$ at cryogenic temperatures. In practice, a coupling of between 0.1 and 1.0 was obtained at $5 \mathrm{~K}$ due to uncertainty in the prediction of the transducer $Q$ prior to cooling.

Apart from having a high sensitivity, a transducer for a resonant bar antenna must also not significantly degrade the acoustic $Q$ of the antenna, or couple vibrations from the experimental chamber to the antenna. Previously, the re-entrant cavity transducer for the 1.5 -tonne $\mathrm{Nb}$ antenna was magnetically levitated and servocontrolled to its operating position near the bending flap. ${ }^{6,7}$ The operating position was determined by the fixed frequency of a high $Q$ sapphire-loaded superconducting cavity resonator (SLOSC).${ }^{10}$ Unfortunately, residual low-frequency motion of the transducer caused the power incident on the cryogenic GaAs metal-semiconductor field-effect transistor (MESFET) preamplifier to vary sufficiently that the noise temperature of the amplifier was degraded. ${ }^{11}$ The noise was attributed to flicker noise which was enhanced by nonlinearities in the amplifier. To reduce the low-frequency motion of the transducer to an acceptable level, a prohibitive increase in the transducer servocontrol loop gain was required.

To circumvent this problem, the re-entrant cavity 
transducer is now bonded to the base of the bending flap with a thin layer of epoxy resin (24-h Araldite) as shown in Fig. 1. Experiments in bonding low acoustic loss niobium components indicated that the bonded re-entrant cavity should not significantly degrade the acoustic $Q$ of the antenna. ${ }^{12}$ An acoustic $Q$ of $1.7 \times 10^{7}$ has been achieved for the composite antenna, consisting of niobium bar, bonded bending flap, and bonded transducer. ${ }^{13}$ This $Q$ value was limited by gas damping of the bending flap. The intrinsic $Q$ of the antenna is expected to be about $5 \times 10^{7}$.

Although a precise and reproducible method of bonding the transducer to the antenna was devised, ${ }^{5}$ it was not possible to bond it so that its frequency coincided exactly with that of the SLOSC. Since it is difficult to devise a method for fine tuning the cavity frequency that does not degrade the $Q$ of the antenna or couple vibrations to the antenna, it was therefore necessary to have a tunable ultralow phase noise microwave source. For this purpose, a tunable sapphire-loaded superconducting cavity ( $\mathrm{T}$ SLOSC) is being developed in our laboratory. ${ }^{5,14,15}$ The T-SLOSC consists of a niobium cavity which is loaded with a sapphire mushroom and a thin sapphire disk which can be moved so as to tune the resonant frequencies of the cavity. The very low loss of the sapphire enables extremely high $Q$ 's $\left(\sim 10^{9}\right)$ to be obtained in this type of resonator. An alternative scheme for obtaining the pump signal for the transducer is to beat a SLOSC loop oscillator with an HP 8662A synthesizer. Both the T-SLOSC and SLOSCHP 8662A pump sources have ultralow levels of phase and amplitude noise. The SLOSC-HP $8662 \mathrm{~A}$ oscillator has a measured phase noise of $-135 \mathrm{dBc} / \mathrm{Hz}$ at $700 \mathrm{~Hz}$ from the pump frequency. ${ }^{16}$ This phase noise figure is equivalent to a $1 \times 10^{-20} \mathrm{~m}$ rms motion of the antenna, which is just above the quantum limit for the 1.5-tonne $\mathrm{Nb}$ antenna.

\section{ELECTRICAL LOSSES IN THE RE-ENTRANT CAVITY}

A superconducting niobium cavity can have very low loss. In our laboratory, electrical $Q$ 's in excess of $10^{9}$ have been achieved at $4.2 \mathrm{~K}$ in cylindrical niobium, and sapphire loaded niobium superconducting cavities with only modest surface preparation. ${ }^{10,17}$ A similar surface preparation was used on the re-entrant cavity transducer, but the $Q$ of the re-entrant cavity was not expected to be as high as that of a cylindrical cavity due to its smaller geometry factor, and due to the very small gap between the cavity re-entrant post and the antenna that enhances its sensitivity to contamination by particulate matter or a dielectric layer. Also, the transducer suffers radiative losses due to the small gap between the transducer and the antenna. The highest unloaded electrical $Q$ achieved in a re-entrant cavity transducer in our laboratory is $6.5 \times 10^{5}$ at $4.2 \mathrm{~K} .{ }^{18}$ This $Q$ is close to the maximum that can be expected at this temperature, as described below, and corresponds to a transducer position bandwidth of $4 \times 10^{-11} \mathrm{~m}$.

The losses in a re-entrant cavity transducer are characterized by the unloaded electrical $Q, Q_{0}$, which is given by $^{17}$

$$
\frac{1}{Q_{0}}=\frac{1}{Q_{m}}+\frac{1}{Q_{r}}+\frac{1}{Q_{d}}
$$

where $Q_{m}^{-1}, Q_{r}^{-1}$, and $Q_{d}^{-1}$ are the magnetic losses, radiative losses, and dielectric losses, respectively. Assuming that the surface resistance of the cavity is uniform, the magnetic losses are given by

$$
Q_{m}=\frac{\Gamma}{R_{s}}
$$

where $R_{s}$ is the superconducting (BCS) surface resistance, and $\Gamma$ is a geometrical factor. The geometrical factor is given by the ratio of the total electromagnetic field energy of the resonant mode of the cavity, to the tangential magnetic field integrated over the surface of the cavity. ${ }^{19}$ For a re-entrant cavity, the expression for $\Gamma$ is

$\Gamma=\mu_{0} \Omega_{0}\left(\frac{\ell \ln \left(r_{2} / r_{1}\right)}{2 \ln \left(r_{2} / r_{1}\right)+\ell\left(1 / r_{0}+1 / r_{2}\right)}\right)$,

where $\Omega_{0}$ is the resonant frequency of the cavity. The reentrant cavity transducer used in the 1.5 -tonne $\mathrm{Nb}$ antenna has $\Gamma=3.63 \times 10^{-4} \mu_{0} \Omega_{0}$.

The BCS surface resistance may be calculated from the Mattis-Bardeen ${ }^{20}$ theory. This theory has been shown to give good agreement with measurements of the surface resistance in niobium cavities at microwave frequencies. ${ }^{21,22}$ Although this microscopic theory is based on relatively few assumptions, the results for the general case are complex and require numerical evaluation. However, for $T$ $<0.5 T_{c}$, the BCS surface resistance at microwave frequencies is an exponential function of temperature; ${ }^{23}$

$$
R_{s} \approx \frac{R_{0} \Omega_{0}^{1.7}}{T} \exp \left(\frac{-\Delta(T)}{k_{B} T_{c}} \frac{T_{c}}{T}\right),
$$

where $T_{c}$ is the superconducting transition temperature, $\Delta(T)$ is the energy gap of the superconductor at temperature $T$, and $R_{0}$ is a constant. In the re-entrant cavity used in the 1.5-tonne $\mathrm{Nb}$ antenna, the calculated magnetic loss at $4.2 \mathrm{~K}$ and $9.8 \mathrm{GHz}$ is $Q_{m} \approx 1.6 \times 10^{6}$.

The radiative losses in a re-entrant cavity transducer are given by

$$
Q_{r}=\frac{\Omega_{0} L_{0}}{R_{r}}
$$

where $L_{0}$ is the inductance of the cavity, and $R_{r}$ is the effective radiation resistance at the outer radius of the cavity. The radiation resistance is a measure of the effectiveness of the rf choke. The choke is based on a shortcircuited half-wavelength transmission line, tuned to 9.8 $\mathrm{GHz}$. The first quarter wavelength is a radial waveguide, while the second quarter wavelength is a coaxial transmission line that is short circuited at the bottom. The effectiveness of the choke is determined by the alignment of the cavity with the sense face of the antenna, and by the tuning of the rf choke to the cavity resonant frequency. Expressions for the radiation resistance were derived by Mann ${ }^{17}$ and Veitch. ${ }^{7}$ The calculated maximum $Q_{r}$ for the present cavity is about $1.4 \times 10^{6}$, which is comparable to the BCS 
losses at $4.2 \mathrm{~K}$ and $9.8 \mathrm{GHz}$. Radiative losses in the cavity should be independent of temperature.

The dielectric losses in a re-entrant cavity are given by

$$
Q_{d}=K x_{0}=\frac{x_{0}}{2 t \epsilon_{f} \tan \delta}
$$

where $t$ is the thickness of the contaminating film on each side of the capacitance gap, $\epsilon_{f}$ is the film's dielectric constant, and $\tan \delta$ is the film's loss tangent. The value of the constant $K$ will depend on the surface preparation of the cavity and the experimental environment. Insufficient cleaning of the cavity surface may leave a residual film of grease or water (ice). The cavity may also become contaminated with dust or ice during assembly and cooling. Dielectric losses in a film formed on the surface of the cavity can easily account for a cavity $Q$ degraded to around $10^{5}$. If the absorbed substance is a polar liquid or gas with $\tan \delta \approx 10^{-2}, \epsilon_{f} \approx 10$, and $t \approx 10^{-9} \mathrm{~m}$, the dielectric loss in a cavity with a gap spacing of $13 \mu \mathrm{m}$ is $Q_{d} \approx 6 \times 10^{4}$. Dielectric losses in an oxide layer on the cavity should be independent of temperature.

\section{ELECTROMECHANICAL COUPLING}

A critical parameter for a resonant bar antenna is the electromechanical coupling between the antenna and the transducer. The coupling is defined as the proportion of the elastic energy of the antenna that can be extracted electrically through the transducer, per cycle. For a re-entrant cavity transducer, the electromechanical coupling may be expressed as ${ }^{7}$

$$
\beta \approx \frac{C_{0} V_{p}^{2} Q_{e}}{2 m_{e} \omega_{a}^{2} x_{0}^{2}},
$$

where $C_{0}$ is the capacitance of the cavity, $V_{p}$ is the peak voltage across the capacitance gap, $Q_{e}$ is the loaded electrical $Q$ of the cavity $\left[Q_{e}=Q_{0} /\left(1+\beta_{e}\right)\right.$, where $\beta_{e}$ is the electrical coupling to the cavity], $m_{e}$ is the effective mass of the antenna (or bending flap), and $\omega_{a}$ is the resonant frequency of the antenna. The peak voltage across the cavity post-antenna gap is given by

$$
V_{p}^{2} \approx \frac{8 Q_{d} \beta_{e} P_{i}}{\Omega_{0} C_{0}\left(1+\beta_{e}\right)^{2}},
$$

so the expression for the electromechanical coupling becomes

$$
\beta \approx \frac{4 \beta_{e} Q_{0}^{2} P_{i}}{m_{e} \omega_{a}^{2} x_{0}^{2} \Omega_{0}\left(1+\beta_{e}\right)^{3}} .
$$

The critical parameters are $Q_{0}$ and $P_{i}$. To achieve a high electromechanical coupling the cavity must have a high electrical $Q$ and be operated at high cavity fields.

The present cavity design is not the optimum design that maximizes the electromechanical coupling between the antenna and the transducer. In a theoretical study of the effect of the dimensions of the cavity on the electromechanical coupling, Mann ${ }^{17}$ showed that a global maximum for $\beta\left(\propto C_{0} Q_{0}\right)$ is reached with a wide re-entrant post $\left(r_{0} \approx r_{2}\right)$. In a wide post cavity, the depth of the cavity

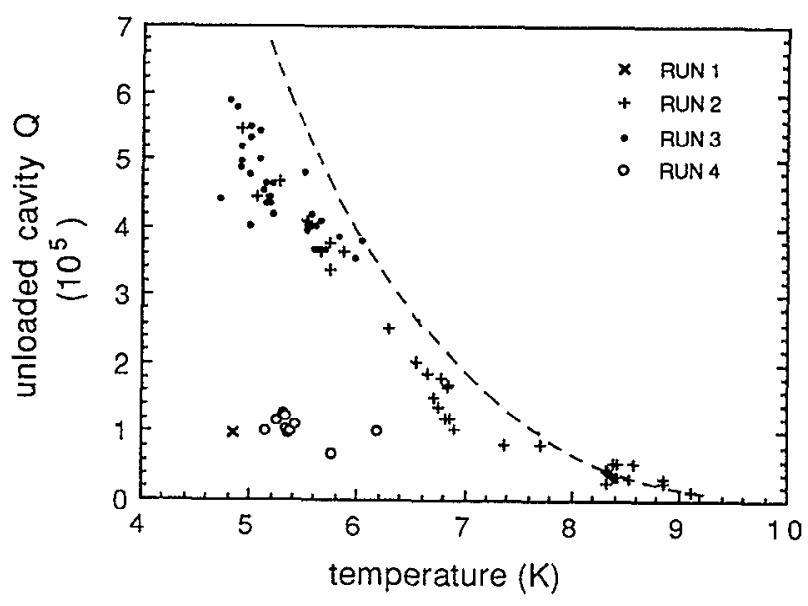

FIG. 3. Temperature dependence of the unloaded electrical $Q$ of the re-entrant cavity transducer. The dashed curve is the calculated BCS surface losses at $9.8 \mathrm{GHz}$.

must be be small for the cavity to be resonant. This increases the radiative losses since the depth of the cavity is no longer large compared to the gap between the transducer and the antenna. Also, increasing the width of the re-entrant post results in greater magnetic losses due to a slight reduction in the cavity geometrical factor. However, the increase in the capacitance of the cavity exceeds the decrease in the cavity $Q$, so the result is a higher electromechanical coupling. Mittoni et al. ${ }^{18}$ showed that the electromechanical coupling for a cavity with a wide re-entrant post $\left(r_{0}=0.8 r_{2}\right)$ could be an order of magnitude greater than that for the present cavity design. In the wide post cavity, the radiative losses limited the $Q$ at $4.2 \mathrm{~K}$ to $1.7 \times 10^{5}$.

\section{TEMPERATURE DEPENDENCE OF THE CAVITY $\boldsymbol{Q}$}

The temperature dependence of the electrical $Q$ of the re-entrant cavity was examined with the transducer bonded to the 1.5-tonne $\mathrm{Nb}$ antenna. The temperature of the antenna was raised above its equilibrium temperature ( $\sim 5 \mathrm{~K}$ ) by allowing all the liquid helium to boil off from the dewar storage tanks. To determine the cavity $Q$, the frequency of the microwave pump was swept slowly across the cavity resonance, and the cavity bandwidth was measured to within $\pm 5 \%$ from the frequency difference between the $3-\mathrm{dB}$ points on the cavity resonance.

The observed temperature dependence of the cavity $Q$ in four antenna runs is shown in Fig. 3. No temperature dependence of the cavity $Q$ was apparent in Runs 1 and 4 as the cavity surface was contaminated. In Runs 2 and 3 , the cavity $Q$ was much higher, and showed a strong dependence on temperature. Below about $6 \mathrm{~K}$, the cavity $Q$ was limited by radiative losses, while at higher temperatures it was degraded by the increase in the BCS surface resistance. Figure 3 also shows the calculated magnetic losses due to the BCS surface resistance at $9.8 \mathrm{GHz}$. The theoretical BCS losses at $8.6 \mathrm{GHz}$ were taken from $\mathrm{Ly}$ neis ${ }^{22}$ and adjusted to the operating frequency of the cavity using Eq. (4). 


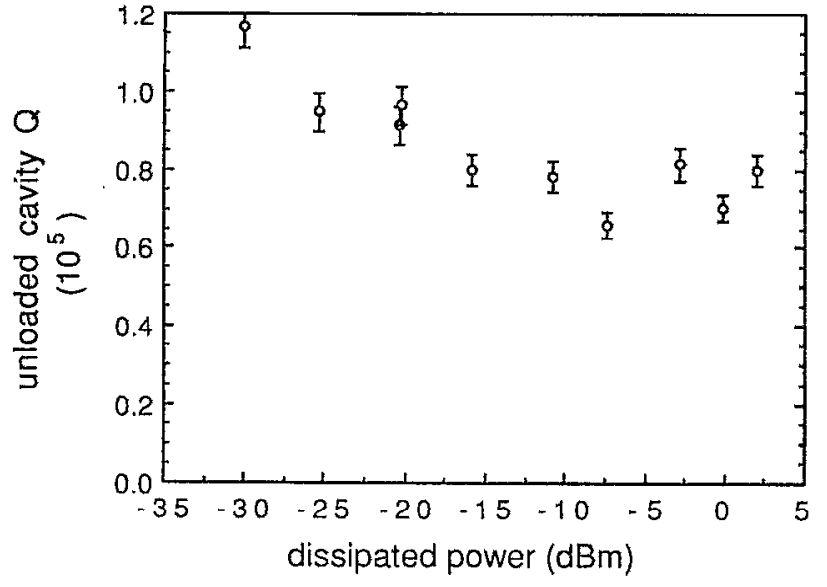

FIG. 4. Dependence of the unloaded electrical $Q$ of the re-entrant cavity transducer on the dissipated power in Run 4.

The highest unloaded $Q$ observed in the re-entrant cavity was $Q_{0}=5.9 \pm 0.3 \times 10^{5}$ in Run 3 at $4.8 \mathrm{~K}$. At this temperature the BCS loss is $Q_{m} \approx 9 \times 10^{5}$. Assuming that dielectric losses were negligible, the calculated radiative loss was then $Q_{r} \approx 1.7 \times 10^{6}$, which is about the same as the calculated maximum $Q_{r}$ for the transducer. Extrapolation of these results indicate that the cavity $Q$ would have been $8.3 \times 10^{5}$ at $4.2 \mathrm{~K}$, and at the superfluid transition temperature $(2.17 \mathrm{~K})$ the $Q$ would have been close to the (radiative loss limited) maximum value.

At liquid-helium temperatures, the resonant frequency of the bonded transducer was found to be temperature dependent. This was due to two effects: the temperature dependence of the superconducting penetration depth of the cavity walls, and thermal contraction of the (nonuniform) cavity-flap epoxy bond. At $5 \mathrm{~K}$ the temperature coefficients of these two effects were -10 and $-40 \mathrm{kHz}$ / $\mathrm{K}$, respectively. It is expected that the temperature coefficient of the cavity-flap bond could be significantly reduced by using a small set of glue pads on the base of the transducer instead of a single bonding surface. Even so, for a transducer with a loaded cavity $Q$ of $5 \times 10^{5}$, the temperature stability of the antenna must be better than a few millikelvins if the resonant frequency of the cavity is not to drift by more than $1 \%$ of the bandwidth.

\section{POWER DEPENDENCE OF THE CAVITY $Q$}

In Run 4 the unloaded cavity $Q$ was almost independent of the power dissipated in the cavity (see Fig. 4). It was expected that any increase in the BCS losses caused by heating of the cavity would degrade the cavity $Q$. At the highest input power in Run 4, the power dissipatcd in the cavity on the center of the cavity resonance was about 1.7 $\mathrm{mW}$. This level of dissipated power was insufficient to heat the cavity so as to produce a measurable decrease in the cavity $Q$. In this run, the $Q$ of the cavity at low power was only $1.0 \times 10^{5}$. Ferreirinho $0^{24}$ found that even in a high $Q$ re-entrant cavity transducer $\left(Q_{0}=3.2 \times 10^{5}\right)$, the cavity $Q$ was independent of power, for a dissipated power as high as $0.7 \mathrm{~mW}$. It is expected that dielectric breakdown of the

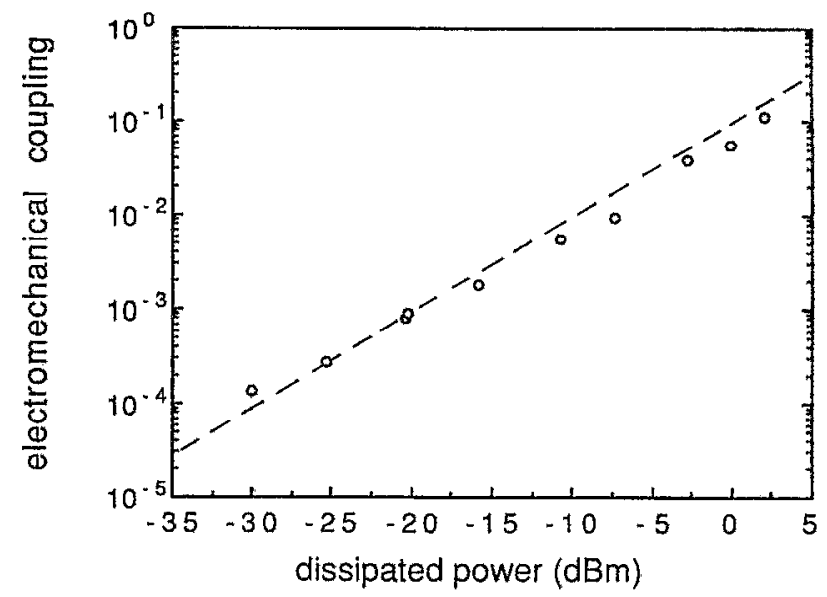

FIG. 5. Dependence of the electromechanical coupling between the antenna and the transducer on the dissipated power in Run 4 . The dashed line is the expected coupling for a cavity $Q$ that is independent of power, with $Q_{0}=1.0 \times 10^{5}$.

exchange gas in the cavity gap would occur before the cavity was heated sufficiently for the cavity $Q$ to be noticeably degraded.

Figure 5 shows a linear increase in the electromechanical coupling with power in Run 4 . The highest coupling achieved was $\beta \approx 0.11$. This coupling surpasses the highest coupling of $\beta=0.054$ reported by Aguiar et al. ${ }^{25}$ for the parametric transducer being developed for the antennae at Louisiana State University. In the present transducer, the maximum coupling was constrained by the available pump power, and not by a failing in the transducer. The maximum available power corresponded to an electric field strength in the cavity-flap gap of $5.5 \mathrm{MV} / \mathrm{m}$. The breakdown strength of vacuum should limit the coupling for this transducer to about unity. If the transducer were to be operated in conjunction with a wideband impedance matching element, an antenna bandwidth comparable to the frequency of the antenna would then be attained.

The power dependence of the cavity $Q$ and electromechanical coupling in Run 1 was reported previously. ${ }^{26}$ In this run, the cavity $Q$ showed a marked degradation with increasing power which limited the coupling at high pump power. The cavity was probably contaminated by dust or ice in the cavity-flap gap.

\section{DISCUSSION}

A re-entrant cavity parametric transducer system for a resonant bar antenna is more intricate than a passive transducer system, such as that based on a dc superconducting quantum interference device (SQUID). First, there is the need to obtain a pump signal that has ultralow levels of phase and amplitude noise. Also, with a high-sensitivity parametric transducer there is a complex interaction between the transducer and the antenna. This phenomenon, which is pronounced at high coupling, has been intensively studied ${ }^{4,13,27,28}$ with the aim of determining the optimum operating conditions for the transducer.

The sensitivity of a resonant bar antenna is indicated by the product $\beta Q_{d} / T_{t}$ where $Q_{t}$ is the loaded acoustic $Q$ 
of the antenna, and $T_{r}$ is the loaded mode temperature. ${ }^{29}$ The loaded mode temperature and $Q$ must be considered because the transducer may significantly affect these quantities. Due to the coupling between the antenna and the transducer, electrical losses in the transducer resistively damp the antenna, decreasing the antenna $Q$, and thus increasing the thermal noise in the antenna. To obtain a very high electromechanical coupling with a passive transducer, the electrical $Q$ of the transducer must be comparable to the acoustic $Q$ of the antenna. This is a very stringent requirement, and in practice the antenna may need to be cooled to $\mathrm{mK}$ temperatures to compensate for the degradation of the antenna $Q$ at high coupling.

One of the advantages of a parametric transducer over a passive transducer is that a high coupling may be used without significant resistive damping of the antenna. In a parametric transducer operating at a much higher frequency than the frequency of the antenna, the effect of electrical noise is reduced. Also, parametric transducers reactively cold-damp the antenna, changing both the mode temperature and the $Q$ of the antenna. ${ }^{4,30}$ Provided that the noise temperature of the transducer $T_{e}$ is sufficiently small, the ratio of the mode temperature to acoustic $Q$ remains almost unchanged. In contrast to a passive transducer, a parametric transducer does not then affect the thermal noise in the antenna.

To achieve noiseless damping in the 1.5-tonne $\mathrm{Nb}$ antenna at moderately high coupling, say $\beta \approx 10^{-3}$, the transducer must have $T_{e}<50 \mathrm{mK}$. This requirement is readily achievable in a superconducting re-entrant cavity transducer. ${ }^{31}$ Unfortunately, the requirement on the noise temperature of the parametric transducer increases as the coupling increases. At the maximum coupling achieved in the 1.5 -tonne $\mathrm{Nb}$ antenna $(\beta \approx 0.11)$, the transducer must have $T_{e}<0.5 \mathrm{mK}$. The previously reported measurement of the transducer noise temperature was limited by excess mechanical vibrations internal to the antenna dewar, with the pump oscillator and demodulator not contributing significantly to the result. The antenna dewar is presently being rebuilt to improve the mechanical isolation of the antenna. ${ }^{32}$ In the next run, the transducer noise temperature will be measured at high coupling. It is known that the phase and amplitude noise of the pump oscillator is not significantly degraded at high output power.

The results reported in this paper indicate that the product, $\beta Q_{d} T_{h}$, can be very high for an antenna using a re-entrant cavity transducer. Combining the measured acoustic $Q$ for the 1.5-tonne $\mathrm{Nb}$ antenna with the highest coupling, gives $\beta Q_{d} / T_{\ell} \approx 4 \times 10^{5}$. This can be compared with $\beta Q d T$, values of 70 for the Rome antenna at CERN, and $3.8 \times 10^{3}$ for the Stanford antenna. ${ }^{29}$

A potential problem with a high $Q$ transducer in a resonant bar antenna is that the interaction between the antenna and the transducer may produce additional noise by changing the vibration amplitude of the antenna. ${ }^{13,32}$ Large amplitude low-frequency seismic noise may modulate the resonant frequency of the cavity, thereby changing the frequency offset of the pump from the center of the cavity resonance, and hence modulating the antenna mode temperature. The vibration amplitude of the antenna mode then increases or decreases in response to the change in the mode temperature, with the rate of attainment of thermal equilibrium determined by the loaded $Q$ of the antenna. Clearly, the stability of the pump frequency offset must be such that the "seismic modulation" noise is less than that expected due to Brownian motion. The seismic modulation noise contribution is power dependent and may therefore limit the achievable electromechanical coupling in the antenna. The antenna suspension and the vibration isolator for the microwave cable to the transducer must be designed to attenuate low-frequency seismic noise, as well as vibrations at the antenna frequency. As an additional measure, a pump frequency servosystem may be implemented to follow the changes to the cavity frequency.

To prevent seismic noise from coupling from the experimental chamber to the transducer, a method of measuring the vibration amplitude of the antenna without any wires or cables attached to the antenna is being developed. ${ }^{32}$ This noncontacting arrangement uses a pair of niobium microwave horns to couple the signal to the transducer.

In summary, a re-entrant cavity parametric transducer is a practical type of transducer for a cryogenic resonant bar gravitational radiation antenna. This type of transducer may have a very high electrical $Q$ and be operated at high cavity fields. An electromechanical coupling of almost unity may be achieved, allowing the antenna to have a high sensitivity and large bandwidth.

\section{ACKNOWLEDGMENTS}

This work was done as part of the Gravitational Radiation Detection Project at the University of Western Australia. The research was supported by the Australian Research Council. The authors would like to acknowledge the contributions of Peter Veitch, Tony Mann, and John Ferreirinho.

${ }^{1}$ V. B. Braginsky, V. I. Panov, and V. D. Popel'nyuk, JETP Lett. 33, 405 (1981).

${ }^{2}$ W. C. Oelfke and W. O. Hamilton, Rev. Sci. Instrum. 54, 410 (1983).

${ }^{3}$ K. Tsubono, M. Ohashi, and H. Hirakawa, Jpn. J. Appl. Phys. 25, 622 (1986).

${ }^{4} \mathrm{P}$. J. Veitch, in The Detection of Gravitational Radiation, edited by D. G. Blair (Cambridge University Press, Cambridge, 1991), pp. 186-225.

${ }^{5}$ P. J. Veitch, D. G. Blair, N. P. Linthorne, L. D. Mann, and D. K. Ramm, Rev. Sci. Instrum. 58, 1910 (1987).

${ }^{6}$ D. G. Blair and A. G. Mann, Nuovo Cimento B 61, 73 (1981).

${ }^{7}$ P. J. Veitch, Ph.D. thesis, University of Western Australia 1986.

${ }^{8} \mathrm{~K}$. Fujisawa, IRE Trans. Microwave Theory Tech. 6, 344 (1958).

${ }^{9}$ V. V. Kolesov, V. I. Panov, and V. G. Petnikov, Cryogenics 17, 299 (1977).

${ }^{10}$ D. G. Blair and S. K. Jones, J. Phys. D 20, 1559 (1987).

"L. D. Mann, D. G. Blair, and K. J. Wellington, Electron. Lett. 22, 1037 (1986).

${ }^{12}$ D. G. Blair and D. K. Ramm, Cryogenies 26, 322 (1986).

${ }^{13}$ N. P. Linthorne, Ph.D. thesis, University of Western Australia, 1991.

${ }^{14}$ M. E. Tobar and D. G. Blair, IEEE Trans. Microwave Theory Tech. MTT-39, 1582 (1991).

${ }^{15}$ M. E. Tobar and A. G. Mann, IEEE Trans. Microwave Theory Tech. MTT-39, 2077 (1991).

${ }^{16}$ D. G. Blair, N. P. Linthorne, A. G. Mann, Peng Hong, K. M. Sebo, M. E. Tobar, and P. J. Turner, in Gravitational Astronomy: Instrument Design and Astrophysical Prospects, edited by D. E. McClelland and H. 
A. Bachor (World Scientific, Singapore, 1991), pp. 172-188.

${ }^{17}$ A. G. Mann, Ph.D. thesis, University of Western Australia, 1982.

${ }^{18}$ L. J. Mittoni, A. G. Mann, N. P. Linthorne, and D. G. Blair, J. Phys. D (submitted).

${ }^{19}$ E. Maxwell, in Progress in Cryogenics, edited by K. Mendelssohn (Heywood, London, 1964), Vol. 4, pp. 124-158.

${ }^{20}$ D. C. Mattis and J. Bardeen, Phys. Rev. 111, 412 (1958).

${ }^{21}$ J. P. Turneaure and I. Weissman, Appl. Phys. 39, 4417 (1968).

${ }^{22}$ C. M. Lyneis, Ph.D. thesis, Stanford University 1974, pp. 40, 89.

${ }^{23}$ H. Pfister, Cryogenics 16, 17 (1976).

${ }^{24}$ J. Ferreirinho (private communication).

${ }^{25}$ O. D. Aguiar, W. W. Johnson, and W. O. Hamilton, Rev. Sci. Instrum. 62, 2523 (1991).

${ }^{26}$ N. P. Linthorne, P. J. Veitch, D. G. Blair, P. J, Turner, M. E. Tobar, and A. G. Mann, Physica B 165, 9 (1990).

${ }^{27}$ N. P. Linthorne, P. J. Veitch, and D. G. Blair, J. Phys. D 23, 1 (1990).

${ }^{28}$ M. E. Tobar, N. P. Linthorne, and D. G. Blair, in Gravitational Astron- omy: Instrument Design and Astrophysical Prospects, edited by D. E. McClelland and H. A. Bachor (World Scientific, Singapore, 1991), pp. 368-378.

${ }^{29}$ P. J. Veitch, D. G. Blair, M. J. Buckingham, C. Edwards, N. P. Linthorne, L. D. Mann, N. P. Prestage, D. K. Ramm, and M. Zeng, in Experimental Gravitational Physics, edited by P. F. Michelson (World Scientific, Singapore, 1988), pp. 353-357.

${ }^{30}$ H. Hirakawa, S. Hiramatsu, and Y. Ogawa, Phys. Lett. A 63, 199 (1977).

${ }^{31}$ M. E. Tobar, D. G. Blair, N. P. Linthorne, and P. J. Turner, in Proceedings of the Sixth Marcel Grassmann Meeting on General Relativity (to be published, 1992).

${ }^{32}$ D. G. Blair, P. J. Turner, D. Berinson, Ju Li, F, van Kann, N. P. Linthorne, A. G. Mann, Peng Hong, and M. E. Tobar, in Proceedings of the Sixth Marcel Grossmann Meeting on General Relativity (to be published, 1992). 
Review of Scientific Instruments is copyrighted by the American Institute of Physics (AIP). Redistribution of journal material is subject to the AIP online journal license and/or AIP copyright. For more information, see http://ojps.aip.org/rsio/rsicr.jsp Copyright of Review of Scientific Instruments is the property of American Institute of Physics and its content may not be copied or emailed to multiple sites or posted to a listserv without the copyright holder's express written permission. However, users may print, download, or email articles for individual use. 